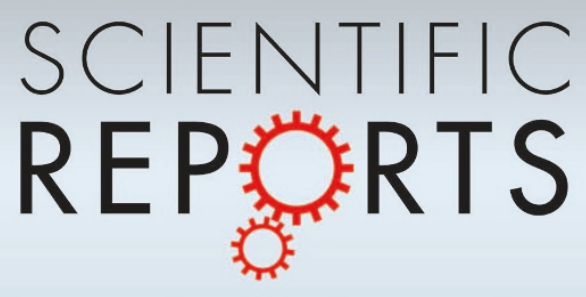

OPEN

SUBJECT AREAS:

NUTRITIONAL

SUPPLEMENTS

MOLECULAR ENGINEERING IN PLANTS

Received

11 September 2013

Accepted

7 January 2014

Published

27 January 2014

Correspondence and requests for materials should be addressed to S.-T.H. (seonghong@ chonbuk.ac.kr)

* These authors contributed equally to this work.

\section{Treatment of obesity with the resveratrol-enriched rice DJ-526}

\author{
So-Hyeon Baek ${ }^{1 *}$, Hea-Jong Chung ${ }^{2 *}$, Heui-Kwan Lee ${ }^{2 *}$, Roshan D'Souza ${ }^{2}$, Youngju Jeon ${ }^{2}$, \\ Hyeon-Jin Kim ${ }^{3}$, Soon-Jong Kweon ${ }^{4} \&$ Seong-Tshool Hong ${ }^{2}$
}

\begin{abstract}
${ }^{1}$ National Institute of Crop Science, Rural Development Administration, Iksan, Chonbuk, Korea, ${ }^{2}$ Department of Biomedical Sciences, Chonbuk National University Medical School, Jeonju, Chonbuk, Korea, ${ }^{3}$ JINIS BDRD institute, JINIS Biopharmaceuticals Co., 948-9 Dunsan, Bongdong, Wanju 565-902, Jeollabuk-do, South Korea, ${ }^{4}$ National Academy of Agricultural Science, Rural Development Administration, Suwon, Gyeonggi, Korea.
\end{abstract}

Obesity is the most prevalent disease in the world which poses a serious risk for various chronic diseases. However, currently there are not any therapeutic agents that reduce body weight without causing serious side effects. In order to prevent and/or treat obesity and related diseases through a nutraceutical approach, we created a resveratrol-enriched transgenic rice accumulating $1.4 \mu \mathrm{g} / \mathrm{g}$ of resveratrol in its grain, DJ-526. Feeding of mice with the resveratrol-enriched rice DJ-526 showed excellent anti-obesity effect with reduction of body weights and abdominal fat volumes compared to the control by $20.0 \%$ and $31.3 \%$, respectively. Also, the consumption of the resveratrol-enriched rice DJ526 significantly improved the blood lipid profiles and glucose levels in the animal experiments. Our resveratrol-enriched rice DJ-526 rice could provide both safe and convenient way for people with obesity and related diseases without major change of lifestyle or unwanted side effects from medication.

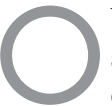

besity is a major health problem throughout the world, and it is increasing both in prevalence and severity ${ }^{1}$. According to the $\mathrm{WHO}$, the current population of 1.6 billion overweight/obese people is expected to double by 2015. Pharmaceutical drugs such as orlistat, lorcaserin, sibutramine, phentermine, diethylpropion, or fluoxetine have been developed for the treatment of obesity ${ }^{2,3}$. However, benefits of these pharmaceutical drugs do not outweigh the side effects of the drugs in most cases ${ }^{4}$. Treatments of obesity with these drugs despite short-term benefits, is often associated with rebound weight gain after the cessation of drug use and serious side effects from the medication. As such, it is necessary to develop a new type of anti-obesity treatment that treats obesity without long-term side effects. Because obesity is caused by a nutrient-uptake imbalance where the amount of energy intake exceeds the amount of energy expended, one option to treat obesity effectively without side effect would be a nutraceutical approach ${ }^{5,6}$. Although some have been described to have dietary effects, the impact on obesity and related diseases are not enough to be claim its efficacy ${ }^{7}$. For an effective treatment, it would be reasonable to think that supplementation of nutrient ingredients having anti-obesity effect to an anti-obesity nutraceutical would generate a much more fortified anti-obesity nutraceutical.

Resveratrol (3,5,4' -trihydroxy-trans-stilbene) has been well recognized for its lipid-lowering function as well as calorie-restriction effect, proposing itself as one of the best anti-obese ingredients ${ }^{8-11}$. To generate more effective and convenient way for dietary consumption of resveratrol other than red wine, one of the major research efforts in plant science was to create transgenic cereal plants that accumulate resveratrol in their grains ${ }^{12,13}$. However, resveratrol was only detected at low levels in the leaves and stems except our previous work ${ }^{14}$. We previously created the resveratrol-enriched rice by transferring the resveratrol biosynthesis gene, stilbene synthase (STS) to Dongjin rice through the expression validation approach ${ }^{15}$. Among the $398 \mathrm{~T}_{1}$ small plantlets, one line of resveratrol-enriched rice plants, DJ-526, showed the excellent agricultural characteristic as well as accumulating a large quantity of resveratrol in its grain.

Parental plant of the resveratrol-enriched rice DJ-526, Oryza sativa japonica variety Dongjin rice developed by the Rural Development Administration of Korea, has characteristics of having grains rich in fiber and polyphenols with anti-obesity activity ${ }^{14}$. Thus, the resveratrol-enriched rice DJ-526 accumulating a large quantity of resveratrol in its grains in addition to fiber and polyphenols could be an ideal nutraceutical to treat obesity and its related diseases. The purpose of this research was to investigate whether the resveratrol-enriched rice DJ-526 is effective for obesity and its related diseases through a synergistic combination of the innate anti-obesity property of Dongjin rice and the lipid-lowering property of transgenic resveratrol. Our animal experiments showed that 
the resveratrol-enriched rice DJ-526 has strong anti-obesity effects and significantly improved all aspects of obesity-related diseases, suggesting its potential as an anti-obesity nutraceutical.

\section{Results}

The grains of the resveratrol-enriched rice DJ-526 contained a large amount of resveratrol. One of the current challenges in creating transgenic plants is to make a desired gene or genes functional specifically in the targeted part of the transgenic plants. It has been well known that piceid are accumulated as one of the major compounds in the plants having either natural or transgenic resveratrol biosynthetic pathways ${ }^{16}$. Because the health benefits of piceid are insignificant and long-term effect on the physiology of human has not been completely understood ${ }^{17,18}$, we aimed to select a transgenic rice plant accumulating mostly resveratrol rather than piceid in its grain. As we previously reported ${ }^{15}$, we first massively screened the resveratrol quantity of $398 \mathrm{~T}_{1}$ transgenic plants which contained the AhSTS1gene for resveratrol production. After identifying the candidate $\mathrm{T}_{1}$ transgenic plants, the candidate $\mathrm{T}_{1}$ transgenic plants were transplanted into a rice paddy. By the thorough analyses of the metabolic profile of the resveratrol and its related metabolites in the every portion of the transgenic plants, we selected a transgenic plant accumulating large quantity of resveratrol in its grain, DJ-526 $6^{15}$. Figure 1 showed the metabolic profiles of
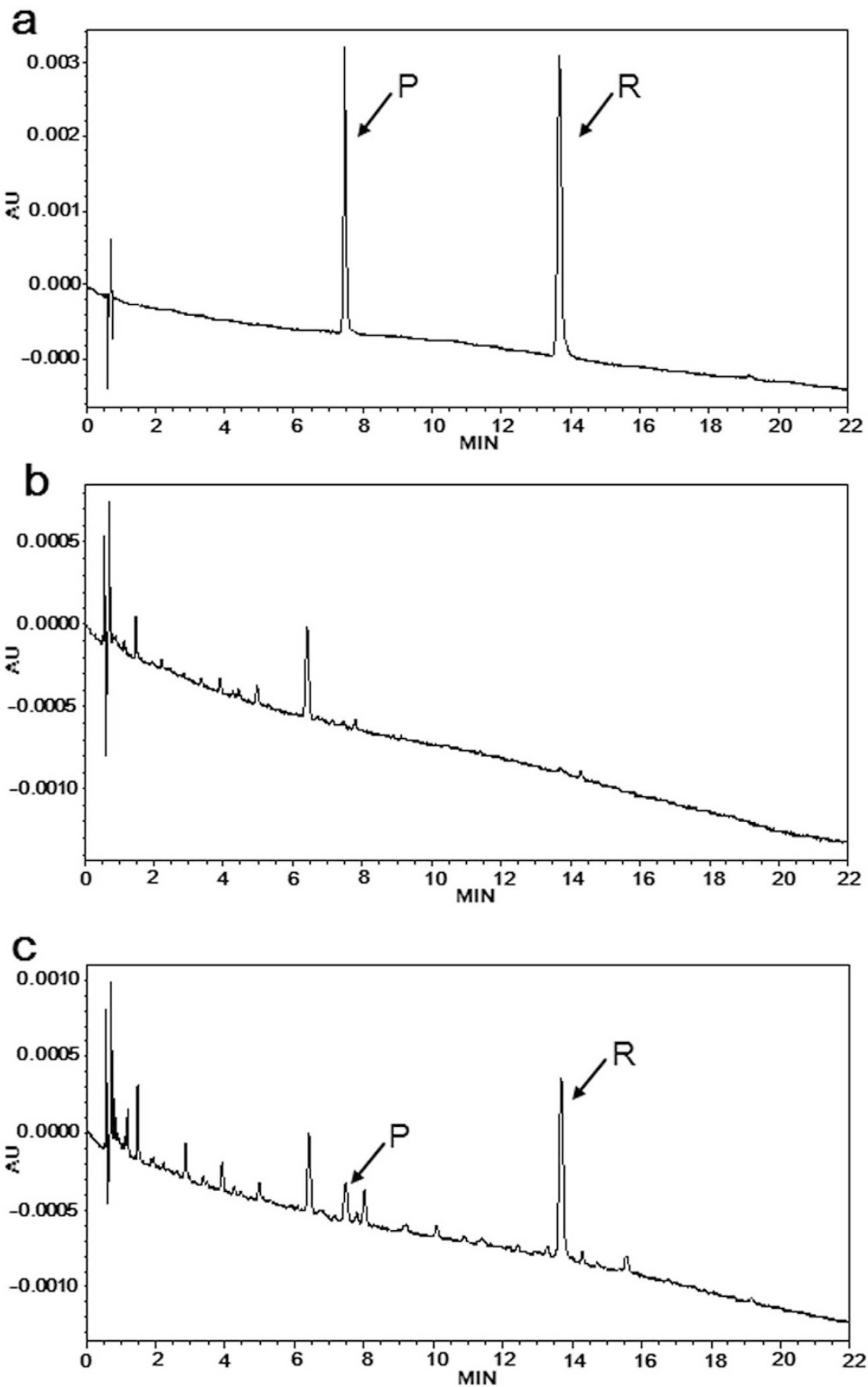

Figure 1 Identification of resveratrol and piceid in the grains of wild type Dongjin and the resveratrol-enriched rice DJ-526 by HPLC. (a) The HPLC profile of a standard mixture of piceid and resveratrol. (b) The HPLC profile of the grains of wild type Dongin. (c) The HPLC profile of the grains of the resveratrol-enriched rice DJ-526. Arrows indicate piceid (P) and resveratrol (R) that correspond to each standard. 

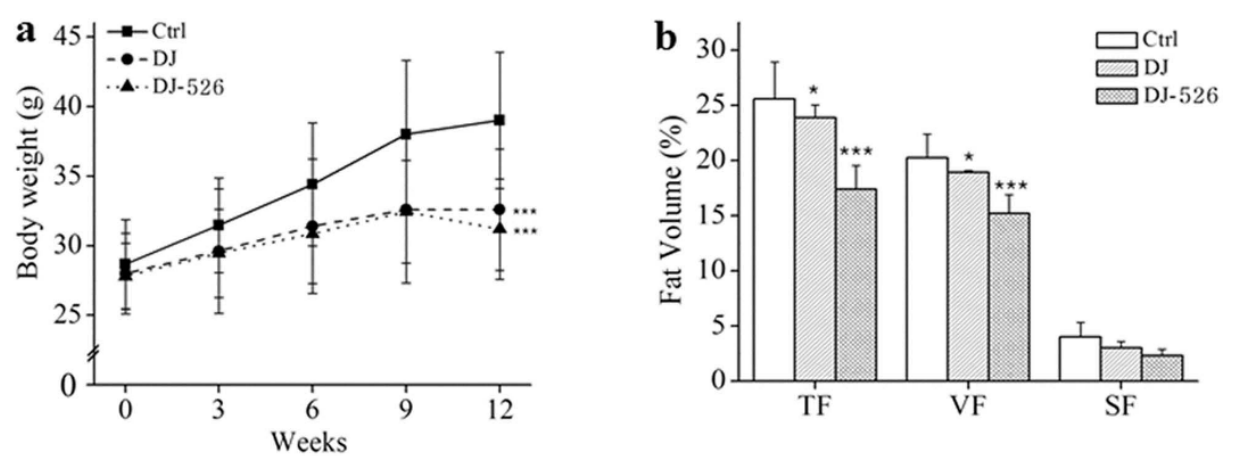

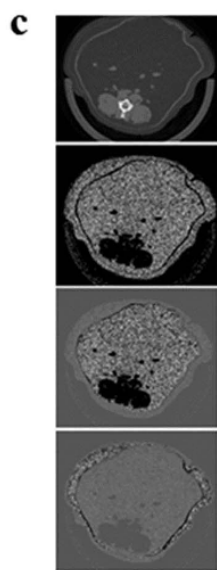

Ctrl

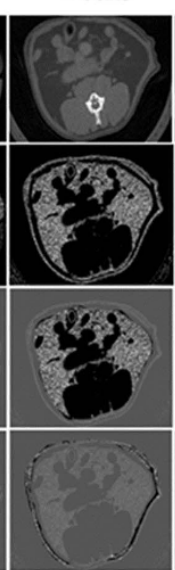

DJ

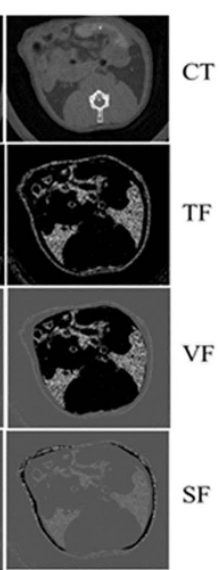

DJ-526

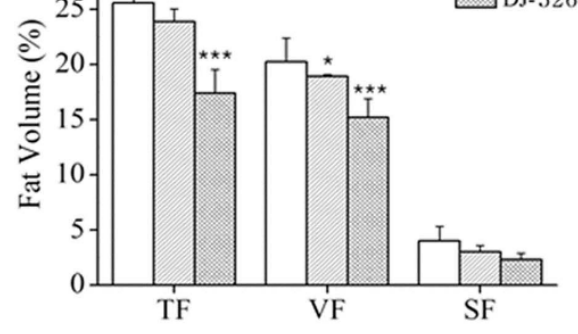

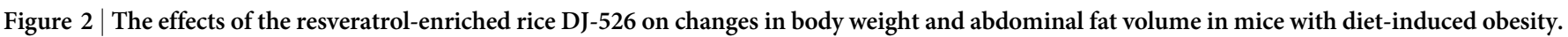

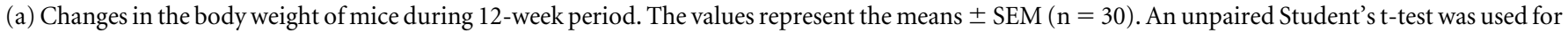
the statistical analysis; ${ }^{* *} \mathrm{p}<0.001$ compared with Ctrl. (b) Changes in the fat volume of mice measured by in vivo micro-CT image analysis.

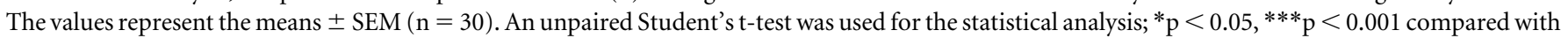
Ctrl. (c) The representative images of the micro-CT image analysis. TF, total fat; VF, visceral fat; SF, subcutaneous fat.

resveratrol and the its related compound, piceid, from the resveratrol-enriched rice DJ-526 using HPLC. As expected, neither resveratrol nor piceid was detected in the HPLC analysis on the wild type Dongjin rice (Figure 1b). On the other hand, the grains of the resveratrol-enriched rice DJ-526 contained a relatively high quantity of resveratrol compared with piceid (Figure 1c), whereas the high quantity of piceid compared with resveratrol was observed in the leaves. In fact, resveratrol quantity in the grain of the resveratrolenriched rice DJ-526, $1.4 \mu \mathrm{g} / \mathrm{g}$, were close to the typical levels of resveratrol quantity in high-quality red wine, $0.8-5.8 \mu \mathrm{g} / \mathrm{mL}^{19}$. The preferential distribution of the two related metabolites, high accumulation of resveratrol in the edible grains but low in the leaves, makes the resveratrol-enriched rice DJ-526 ideal as a resveratrol-enriched cereal plant.

The resveratrol-enriched rice DJ-526 rice showed an excellent antiobesity effect by reducing body weights and abdominal fat volumes. To investigate whether the resveratrol-enriched rice DJ526 has an anti-obesity effect through a synergistic effect of the innate characteristics of Dongin and the transgenic resveratrol as we expected, the efficacy of the resveratrol-enriched rice DJ-526 on obesity was examined using an in vivo mouse model. The C57BL/6 inbred mice with diet-induced obesity were fed the high fat diet (HFD) for 12 weeks in the control group or a modified HFD in the experimental group, in which the carbohydrate source was replaced with either Dongjin rice or the resveratrol-enriched rice DJ-526 (supplementary Table S1). Because body weight and abdominal fat volumes are the phenotypes of obesity, changes in the body weight and abdominal fat volumes in each mouse group were periodically monitored under the continued HFD conditions. The food consumption rate was the same among different mouse groups during the experimental period. Figure 2 displayed the changes in the body weight and abdominal fat volumes after dietary consumption of the resveratrol-enriched rice DJ-526. As shown in figure $2 \mathrm{a}$, body weights were greatly reduced in mice fed with the resveratrol-enriched rice DJ-526 by $20.0 \%$ compared to the control, slightly greater than Dongjin rice group. More significant data came from micro-CT image analyses on abdominal fat deposition (Figure 2b). The total, visceral and subcutaneous fat volumes in the resveratrol-enriched rice DJ-526 group were $17.4 \%, 15.2 \%$ and $2.3 \%$, respectively, which were significantly lower than the fat volumes from the control $(25.6 \%, 20.2 \%$ and $4.0 \%$, respectively) and the Dongjin group $(23.9 \%, 18.9 \%$ and $3.0 \%$, respectively). The representative images of the micro-CT image analyses on abdominal fat deposition clearly indicated that the total, visceral and subcutaneous fat accumulation was the lowest in the resveratrol-enriched rice DJ526 group compared with the other treatments (Figure 2c). Based on the experimental results of the efficacy of the resveratrol-enriched rice DJ-526 on body weight and abdominal fat volumes, it was concluded that the resveratrol-enriched rice DJ-526 had an excellent anti-obesity effect.

The consumption of the resveratrol-enriched rice DJ-526 significantly improved lipid profiles and blood glucose levels with clear anti-obesity effect in animal experiments under high-fat diet. Obesity is not simply a consequence of overweight but is typically complicated by diabetes and hyperlipidemia. Because the nature of obesity, we examined the efficacy of the resveratrol-enriched rice DJ526 on the obesity-related diseases by monitoring changes in the levels of blood glucose, triacylglycerol, total cholesterol, HDL-cholesterol, 

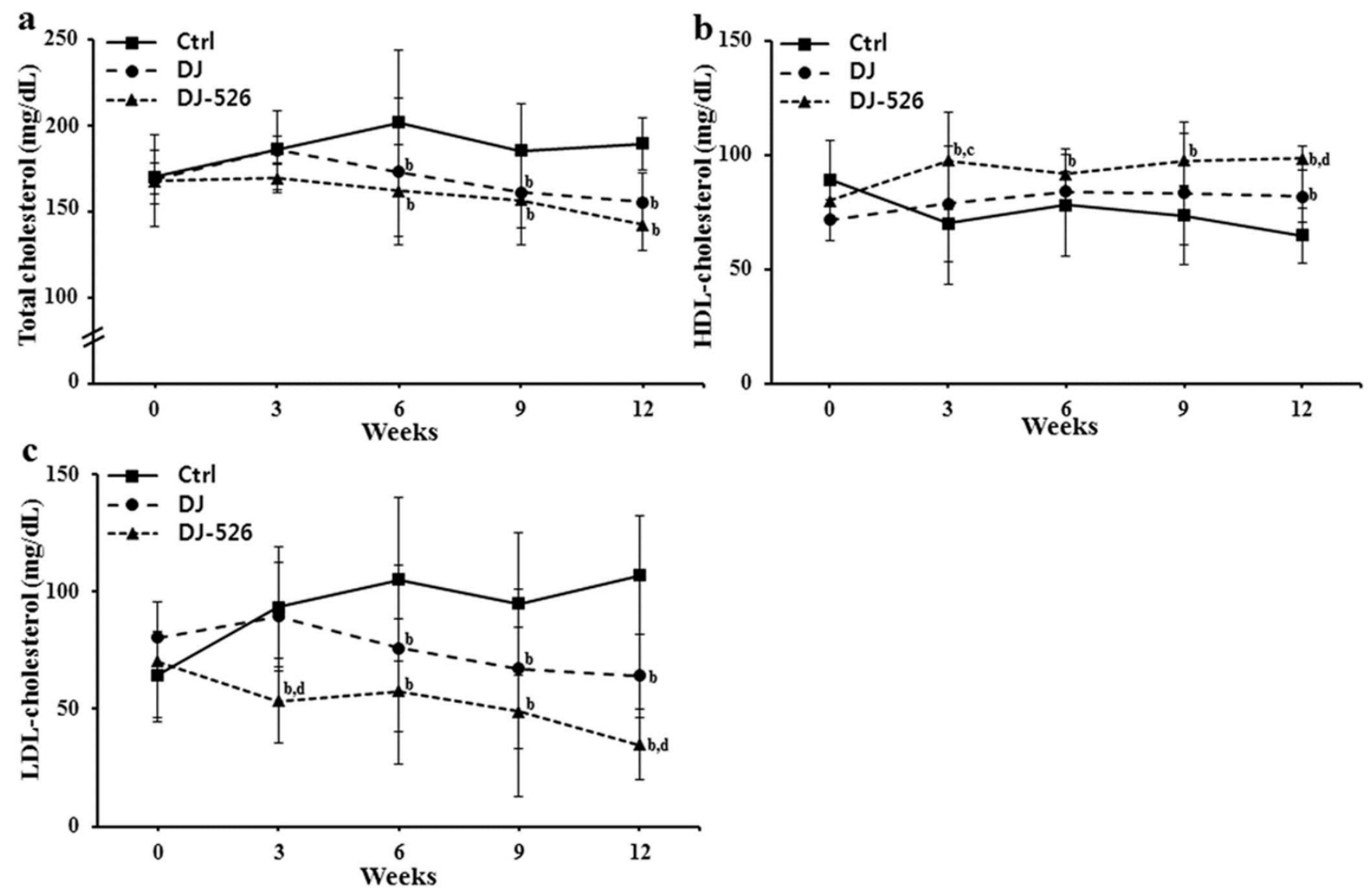

Figure $3 \mid$ The effects of the resveratrol-enriched rice DJ-526 on changes in blood cholesterol profiles in mice with diet-induced obesity. The effects of the resveratrol-enriched rice DJ-526 on blood total cholesterol (a), HDL-cholesterol (b) and LDL-cholesterol (c) of mice during the 12-week experimental period. The values represent the means \pm SEM $(n=30)$. Values in the figure with a superscripted letter indicate statistical significance as analyzed by an unpaired Student's t-test; (a): $\mathrm{p}<0.05$ compared with Ctrl; (b): $\mathrm{p}<0.01$ compared with with Ctrl; (c): $\mathrm{p}<0.05$ compared with Dongin; (d): $\mathrm{p}<0.01$ compared with Dongjin. Ctrl, mice fed a HFD; Dongjin, mice fed a HFD in which the corn starch and sucrose was replaced with Dongjin rice; DJ-526, mice fed a HFD on which the corn starch and sucrose were replaced with the resveratrol enriched rice DJ-526.

LDL-cholesterol using an in vivo mouse model. The consumption of Dongjin rice resulted in some improvements in lipid profile and blood glucose levels compared to the control, as expected from its endogenic nature. More importantly, the consumption of the resveratrolenriched rice DJ-526 significantly improved the lipid profiles as well as blood glucose. The consumption of the resveratrol-enriched rice DJ-526 lowered total cholesterol by $17.9 \%$, and LDL-cholesterol by $67.3 \%$, while increasing HDL-cholesterol by $34.2 \%$ compared to the control (Figure 3). Also, the consumption of the resveratrol-enriched rice DJ-526 lowered blood glucose by $13.9 \%$ and triacylglycerol by $49.3 \%$ compared to the control (Figure 4).

\section{Discussion}

Obesity is complicated with various diseases such as diabetes, hypercholesterolemia, hyperlipidemia, metabolic syndrome, etc ${ }^{1}$ Currently, obesity and its related diseases became a major health problem throughout the world that is increasing both in prevalence and severity ${ }^{20}$. In spite of serious efforts to treat and prevent obesity and its related diseases, an ideal solution for obesity has not been developed. Considering efficacy and their side effects, current pharmaceutical drugs to treat obesity and its related diseases do not provide a solution for obesity and its related diseases so that only $6 \%$ of obese patients are treated pharmacologically ${ }^{21}$. We believe that the resveratrol-enriched rice DJ-526 is an ideal nutraceutical solution to treat or prevent obesity. The anti-obesity effect resulting from the synergistic effect of Dongjin rice and resveratrol showed efficacy levels aiming to treat obesity and its related diseases as much as the typical pharmaceutical drugs. Current pharmaceutical drugs only target individual aspects of obesity and its related diseases, such as blood glucose, LDL/total cholesterol, or body weight. There is no pharmaceutical drug available to treat every aspect of obesity and its related diseases. We believe that the resveratrol-enriched rice DJ-526 could be an ideal choice to target most, if not all, aspects of obesity and its related diseases.

Due to the synergistic effect of the endogenous anti-obesity effect of the Dongjin rice and the lipid-lowering effect of resveratrol, the resveratrol-enriched rice DJ-526 has more potent anti-obesity activity than Dongjin rice itself so that the resveratrol-enriched rice DJ526 can be used to treat and prevent obesity and its related diseases. Both the severity and prevalence of obesity and its related diseases, such as cardiovascular diseases, and diabetes, among many others, are more serious in developing countries than in developed countries. Limited medical care in developing countries make obesity and its related diseases is a more serious issue in developing countries than in developed countries. We believe that the resveratrol-enriched rice DJ-526 could be an excellent alternative for the management of obesity and its related diseases, not only in developed countries but also in developing countries.

Finally our work provides an inspiration for a future development of genetically modified crops. This work showed that a synergistic effect of the innate property of a host plant and a transgenic property significantly augmented the original anti-obesity property of the Dongjin rice. We believe that a careful selection of host plant and transgene would lead to create a very interesting functional crop to manage many chronic diseases. 

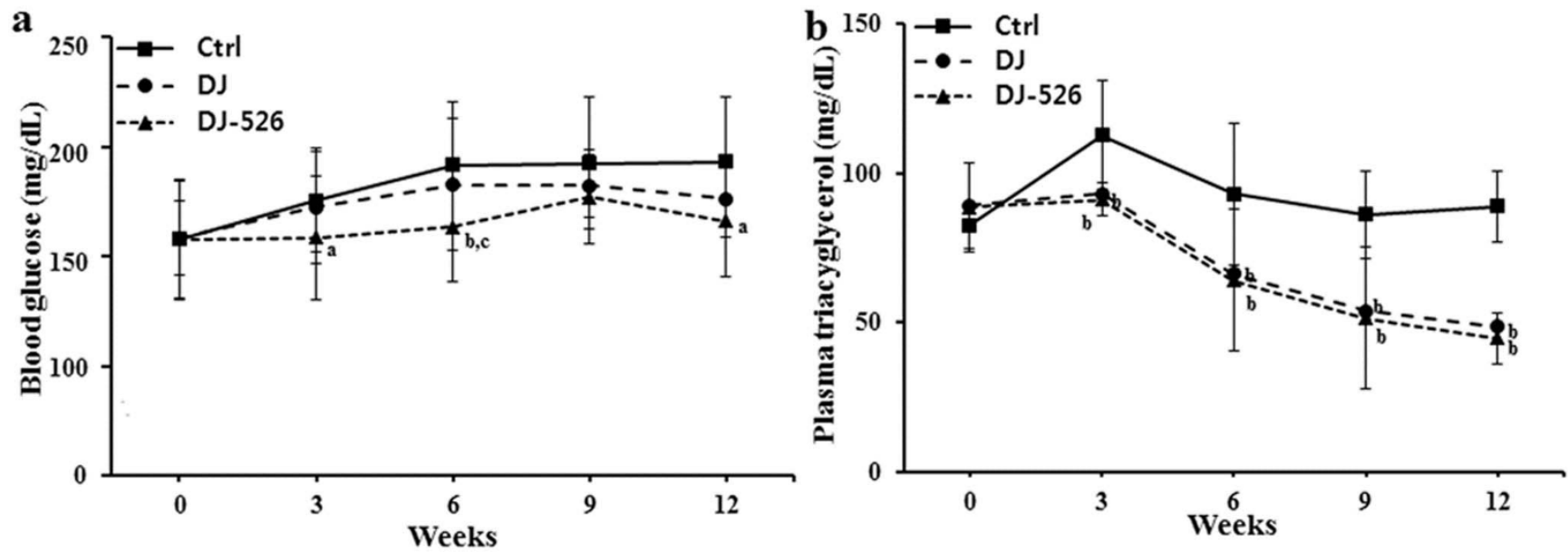

Figure $4 \mid$ The effects of the resveratrol-enriched rice DJ-526 on changes in blood glucose and plasma triacylglycerol in mice with diet-induced obesity. The effects of the resveratrol-enriched rice DJ-526 on the blood glucose (a) and plasma triacyglycerol level (b) of mice during the 12-week experimental period. The values represent the means \pm SEM $(n=30)$. Values in the figure with a superscripted letter indicate statistical significance as analyzed by an unpaired Student's t-test; (a): $\mathrm{p}<0.05$ compared with Ctrl; (b): $\mathrm{p}<0.01$ compared with Ctrl; (c): $\mathrm{p}<0.05$ compared with Dongjin; (d): $\mathrm{p}<0.01$ compared with Dongjin. Ctrl, mice fed a HFD; Dongjin, mice fed a HFD in which the corn starch and sucrose was replaced with Dongjin rice; DJ-526, mice fed a HFD on which the corn starch and sucrose were replaced with the resveratrol enriched rice DJ-526.

\section{Methods}

Quantification of resveratrol and piceid. The eight-week-old leaves and mature grains of the wild-type cultivar Dongjin and the resveratrol-enriched rice DJ-526 rice plants were used to determine the levels of resveratrol and the related resveratrol glucoside piceid as described previously ${ }^{15}$. After the fresh samples of the leaves and grains were freeze-dried, the samples were homogenized to be extracted with $80 \%$ ethanol. $1 \mu \mathrm{L}$ of the each sample was analyzed by a reverse phase HPLC equipped with a UV detector under the gradient condition (ACQUITY TUV, Waters, Milford, USA). The ACQUITY UPLC BEH-C18 $1.7 \mu \mathrm{m}$ column $(2.1 \mathrm{~mm} \times 100 \mathrm{~mm}$, Waters) was used at a flow rate of $0.4 \mathrm{~mL} / \mathrm{min}$. The mobile phase was 10 to $90 \%$ acetonitrile (ACN). A gradient elution was conducted as follows: $0 \mathrm{~min}, 10 \% \mathrm{ACN}$; $1.54 \mathrm{~min}, 10 \% \mathrm{ACN}$; $10 \mathrm{~min}, 15 \% \mathrm{ACN} ; 22 \mathrm{~min}, 25 \% \mathrm{ACN} ; 22.4 \mathrm{~min}, 90 \% \mathrm{ACN}$; and $25 \mathrm{~min}, 90 \% \mathrm{ACN}$; followed by re-equilibration of the column with $10 \% \mathrm{ACN}$ for $5 \mathrm{~min}$ prior to the next injection. Each metabolite peak of samples and standard chemical samples was calculated using the Empower software (Waters) to determine quantity of the metabolites. The HPLC fractions of resveratrol and piceid were further verified using GC-MS analysis with the 6890/5973N GC/MS system (Agilent Technologies, Santa Clara, CA, USA) equipped with an Rtx-5MS capillary column (30 $\mathrm{mm} \times 0.25 \mathrm{~mm}$ I.D., $0.25 \mu \mathrm{m}$ film thickness).

Animal experiments. Six-week-old female C57BL/6 mice (Joongang Experimental Animal Co., Seoul, Korea) were purchased and acclimatized for 2 weeks. Then, the animals were fed with a high-fat diet (HFD) containing $45 \%$ calorie as lard fat (D12451, Research Diets Inc., New Brunswick, NJ, USA) for 12 weeks. Mice with dietinduced obesity and related diseases were randomly divided and fed the HFD, modified HFD in which the corn and sucrose were replaced with Dongjin rice, and modified HFD in which the corn and starch were replaced with the resveratrolenriched rice DJ-526. Food intake and body weight were measured regularly, and blood samples were taken at indicated time points. Fasting blood glucose was measured using Accu-check Glucometer (Roche, Indianapolis, IN, USA) and lipid profile analysis was performed using as enzymatic colorimetric method (Asan Pharm., Yongjin, Korea). Fat volume was analyzed from anesthetized mice by highresolution in vivo micro-CT (Skyscan 1076; Skyscan, Konitech, Belgium). The total fat, visceral fat and subcutaneous fat areas were analyzed with Micro-CT images at the level of the L1-L5 intervertebral disk using CTan Ver.1.10 software (Skyscan).

All animal care and use were performed strictly in accordance with the ethical guidelines by the Ethics Committee of Chonbuk National University Laboratory Animal Center, and the animal study protocol was approved by the institution.

1. Paul, C. et al. The epidemiology of overweight and obesity: public health crisis or moral panic? Int. J. Epidemiol. 35, 55-60 (2006).

2. Ioannides-Demos, L. L., Piccenna, L. \& McNeil, J. J. Pharmacotherapies for Obesity: Past, Current, and Future Therapies. J. Obes. 2011, 179674 (2011).

3. Adan, R. A. Mechanisms underlying current and future anti-obesity drugs. Trends. Neurosci. 36, 133-140 (2013).

4. Kang, J. G. \& Park, C. Y. Anti-Obesity Drugs: A Review about Their Effects and Safety. Diabetes Metab. J. 36, 13-25 (2012)

5. Luís, G. J. et al. Physical activity, energy balance and obesity. Public Health Nutr. 10, 1194-1199 (2007).
6. Shimizu, M., Kubota, M., Tanaka, T. \& Moriwaki, H. Nutraceutical Approach for Preventing Obesity Related Colorectal and Liver Carcinogenesis. Int. J. Mol. Sci. 13, 579-595 (2012)

7. Pittler, M. H. \& Ernst, E. Dietary supplements for body-weight reduction: a systematic review. Am. J. Clin. Nutr. 79, 529-536 (2004).

8. Lagouge, M. et al. Resveratrol improves mitochondrial function and protects against metabolic disease by activating SIRT1 and PGC-1 alpha. Cell 127, 1109-1122 (2006).

9. Baur, J. A. et al. Resveratrol improves health and survival of mice on a high-calorie diet. Nature 444, 337-342 (2006).

10. Rivera, L., Moron, R., Zarzuelo, A. \& Galisteo, M. Long-term resveratrol administration reduces metabolic disturbances and lowers blood pressure in obese Zucker rats. Biochem. Pharmacol. 77, 1053-1063 (2009).

11. Zhuang, H., Kim, Y. S., Koehler, R. C. \& Dore, S. Potential mechanism by which resveratrol, a red wine constituent, protects neurons. Ann. N. Y. Acad. Sci. 993, 276-286 (2003)

12. Leckband, G. \& Lörz, H. Transformation and expression of a stilbene synthase gene of Vitis vinifera L. in barley and wheat for increased fungal resistance. Theor. Appl. Genet. 96, 1004-1012 (1998).

13. Stark-Lorenzen, P. et al. Transfer of a grapevine stilbene synthase gene to rice (Oryza sativa L.). Plant Cell Rep. 16, 668-673 (1997).

14. Choi, H. et al. Comparative nutritional analysis for genetically modified rice, Iksan483 and Milyang204, and nontransgenic counterparts. J. Korean soc. Appl. Biol. Chem. 55, 19-26 (2012)

15. Baek, S. H. et al. Creation of resveratrol-enriched rice for the treatment of metabolic syndrome and related diseases. PLOS One 8, e579302013 (2013).

16. Susanne, R. H. et al. Piceid (Resveratrol Glucoside) Synthesis in Stilbene Synthase Transgenic Apple Fruit. J. Agric. Food Chem. 54, 4633-4640 (2006).

17. Kimura, Y., Okuda, H. \& Arichi, S. Effects of stilbenes on arachidonate metabolism in leukocytes. Biochim. Biophys. Acta. 834, 275-278 (1985).

18. Meng, X. et al. Urinary and plasma levels of resveratrol and quercetin in humans, mice, and rats after ingestion of pure compounds and grape juice. J. Agric. Food Chem. 52, 935-942 (2004).

19. Souto, A. A. et al. Determination of trans-resveratrol concentrations in Brazilian red wines by HPLC. J. Food Comp. Anal. 14, 441-445 (2001).

20. Flegal, K. M., Carroll, M. D., Ogden, C. L. \& Curtin, L. R. Prevalence and trends in obesity among US adults, 1999-2008. JAMA. 303, 235-241 (2010).

21. Lee, J. S., Sheer, J. L., Lopez, N. \& Rosenbaum, S. Coverage of Obesity Treatment: A State-by-State Analysis of Medicaid and State Insurance. Public Health Reports 125, 596-604 (2010).

\section{Acknowledgments}

This work was supported by a grant from the Next-Generation BioGreen 21 program, Rural Development Administration (No.PJ009528) and Technology Development Program for High Value-Added Food, Ministry of Agriculture, Food and Rural Affair (No.313040-03-1-CG000), Republic of Korea. 


\section{Author contributions}

S.B., H.C., H.L., R.D. and Y.J. performed the experiments. H.K., S.K. and S.H. designed experiments, analyzed and interpreted the data, and wrote the manuscript.

\section{Additional information}

Supplementary information accompanies this paper at http://www.nature.com/ scientificreports
Competing financial interests: The authors declare no competing financial interests.

How to cite this article: Baek, S.-H. et al. Treatment of obesity with the resveratrol-enriched rice DJ-526. Sci. Rep. 4, 3879; DOI:10.1038/srep03879 (2014).

(c) (i) This work is licensed under a Creative Commons Attribution 3.0 Unported license. (c) To view a copy of this license, visit http://creativecommons.org/licenses/by/3.0 